\title{
AUTOMATIC NONRIGID MOTION CORRECTION FOR QUANTITATIVE FIRST-PASS CARDIAC MR PERFUSION IMAGING
}

\author{
Mitchel Benovoy ${ }^{\star \dagger}$, Matthew Jacobs $^{\star \S}$, Farida Cheriet ${ }^{\dagger}$, Nagib Dahdah ${ }^{\ddagger}$, Andrew E. Arai ${ }^{\star}$, Li-Yueh Hsu $^{\star}$ \\ ${ }^{\star}$ National Heart, Lung and Blood Institute, National Institutes of Health, Bethesda, USA \\ †Dept. Biomed. Eng., École Polytechnique de Montréal, Montréal, Canada \\ ${ }_{\text {}}$ Dept. Elect. Eng. and Comp. Sci., Catholic University of America, Washington D.C., USA \\ $\ddagger$ Ste-Justine University Hospital Research Center, Montréal, Canada
}

\begin{abstract}
First-pass dynamic contrast-enhanced cardiac magnetic resonance imaging is an increasingly important diagnostic tool for coronary artery disease. It is typically performed with breath holding and electrocardiogram gating to minimize motion, but movement caused by residual respiration, cardiac arrhythmia, or missed gating triggers during image acquisition will induce nonrigid deformations of the myocardium that can hamper interpretation and perfusion quantification. We propose an automatic nonrigid image registration framework to correct motion in a series of cardiac magnetic resonance perfusion images. Our method employs reference frame detection combined with robust flow field estimation using a large displacement optical flow formulation coupled with post-hoc image warping and flow field distortion correction. This framework is multi-threadable and can be applied to standard myocardial series, arterial input function series, and proton density weighted images to facilitate perfusion quantification.
\end{abstract}

Index Terms - cardiac magnetic resonance, perfusion, nonrigid, motion correction

\section{INTRODUCTION}

First-pass dynamic contrast-enhanced cardiac magnetic resonance (CMR) imaging has proven valuable in assessing myocardial perfusion and diagnosing coronary artery disease [1]. Despite increasing sophistication of quantitative CMR analysis tools [2], complex patient movement and nonrigid tissue deformations caused by electrocardiogram gating errors, respiration, or arrhythmia require labor-intensive operator interaction to ensure frame-to-frame correspondence of the myocardial regions of interest $[3,4]$. This manual correction is largely limited to rigid body movement, without which the myocardial time signal intensity curve - the principal CMR perfusion metric - will invariably appear distorted. However, nonrigid movement caused by elastic displacements of the myocardium can hinder qualitative assessment of the per- fusion image series and further impair quantitative analysis, particularly at a pixel-by-pixel level.

Various nonrigid image registration schemes have been introduced to compensate for myocardial motion during CMR perfusion imaging [3]. Notable contributions include the work by Wollny et al. [5], who coupled the quasi-periodicity of respiration motion and independent component analysis to label spatiotemporal perfusion events. These events were used to construct motion-free synthetic reference images to perform nonrigid registration. Similarly, Li et al. [6] constructed a pseudo ground-truth series comprised of rigid body motion correction from which elastic deformations are estimated from ventricular regions of interest (ROI) obtained semi-automatically. Conversely, Tautz et al. [7] used the series' middle frame as a reference for registration with an intensity-insensitive phase-based method coupled with the demons algorithm [8] in a dyadic coarse-to-fine scale-space approach. Finally, Xue et al. [9] presented an optical-flow based approach to consecutively register neighboring images to an automatically determined key frame.

The issues complicating motion correction compiled from this body of work originate from the patient-specific displacement and deformation of different anatomical structures during dynamic contrast enhancement. It is thus difficult to devise population-based statistical models or rely on anatomical assumptions. Furthermore, we note the scarcity of methods correcting motion within auxiliary images used to improve perfusion quantification. These include proton-density (PD) weighted images and dedicated arterial input function (AIF) series. PD images are highly sensitive to the surface coil signal profile and can be used to normalize myocardial signal inhomogeneity prior to perfusion quantification [10], whereas AIF image series are specifically designed to maintain the linearity of the left-ventricle (LV) signal intensity for myocardial blood flow quantification [11]. Hence, automatic image registration capable of handling both rigid and nonrigid movement found in the three aforementioned types of CMR images would be beneficial to increase the accuracy and efficiency of quantitative perfusion image analysis. 


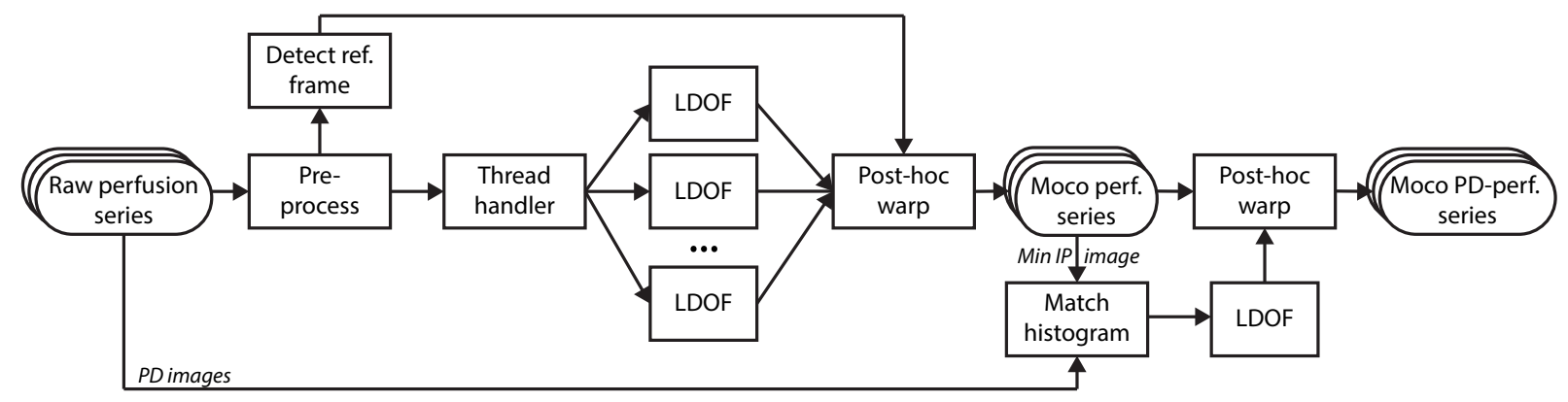

Fig. 1: Automatic nonrigid CMR perfusion image motion correction.

We propose an unsupervised nonrigid motion correction (moco) framework to correct motion in CMR perfusion imaging, as illustrated in Figure 1. Our method employs post-hoc warping following nonrigid displacement estimation using an optical flow formulation specifically amenable to perfusion series dynamics. The novelty of this framework lies in its robustness to all three types of image series (myocardial, AIF, and PD) to facilitate fully quantitative CMR perfusion analysis. Other innovations include a multi-threadable architecture design, automatic reference frame detection, and autonomous flow field distortion correction.

\section{MATERIALS AND METHODS}

The perfusion series included in this study are comprised of 60 frames that were acquired on a 1.5T scanner (Siemens Medical Solutions Inc., Malvern, U.S.A.) during breath hold for 60 heartbeats with a saturation recovery dual-sequence technique [11] based on either steady-state free precession or spoiled gradient echo imaging. For standard T1weighted myocardial perfusion image series (acquisition matrix $=128 \times 80$, image matrix $=256 \times 192$ ), three slices were acquired per heartbeat at the basal, mid-ventricular and apical levels, whereas the AIF perfusion image series (acquisition and image matrix $=64 \times 48$ ) was acquired at the basal location only. Before each perfusion series acquisition, two PD-weighted images were recorded at each slice location.

\subsection{Preprocessing and reference frame identification}

To optimize computation time and accuracy for motion estimation, the raw perfusion image series are first rescaled $(75 \%$ for myocardial images, $150 \%$ for AIF) and quantized to a lower dynamic range. This range compression retains the underlying anatomical structure information, while minimizing noise-prone textural information. A reference frame is then automatically selected and used as a point from which relative motion in the rest of the images is computed. This frame is detected by first denoising the images using PCA decomposition (with $85 \%$ variance retention) to reduce frame-to-frame noise and smooth out structural movement across the time series. The first PCA-reduced frame of the series is then crosscorrelated with its sequential neighbors until a large corre- lation drop is detected, which ostensibly corresponds to the onset of contrast arrival in the right-ventricle (RV). The baseline image immediately preceding this onset is labeled as the reference frame, which depicts contrast-free anatomical structures compared to the later stages, where frame-to-frame pixel intensity fluctuations are higher.

\subsection{Optical flow-based motion estimation and correction}

Starting from the reference image, the pairwise sequentialframe deformation map is computed using an optical flow formulation robust to large displacements (LDOF) [12]. This characteristic is crucial to accommodate the fast contrast arrival without causing significant flow estimation artifacts. The technique employed here merges discrete localized correspondences from histograms of oriented gradients (HOG) descriptor matching [13] with a continuous variational optical flow model. The former handles large displacements of arbitrarily moving anatomical structures, while the latter efficiently computes dense flow fields to correct for subtle deformations such as contractions of the myocardial boundary. The LDOF is adjusted with three parameters: $\alpha$, the level smoothness of the flow field; $\beta$, the weight of the descriptor matching term; and $\gamma$, the weight of the gradient consistency term. These parameters are set to aggressive default values (small $\alpha$, large $\beta \& \gamma$ ) to ensure precise motion estimates, but are modulated automatically to adapt to estimation errors.

Instead of immediately registering the current frame before computing the subsequent motion, the stepwise $x, y$ deformation fields $\left[\mathbf{f}^{\mathbf{x}}, \mathbf{f}^{\mathbf{y}}\right]$ at each image pair were saved for warping at a later stage. Compared to methods that register all images to a single reference frame or to consecutively registered frames, this post-hoc approach has the advantage of avoiding successive smoothing caused by sequential frame-to-frame interpolation and improving the displacement estimation as the motion and appearance between adjacent frames are typically smaller and consistent, respectively. This technique is particularly well-suited for perfusion CMR series that have large motion events due to incomplete patient breath-holding or tissue contrast change at late perfusion. As the estimation of a given flow field does not depend on pre-registered frames, the design of a parallel processing architecture where groups of sequential image pairs are of- 
floaded to multiple processing threads is feasible. Compared to consecutive correction, a $30 \%$ time reduction was measured on a quad-core CPU (Intel Core i7 4770).

Following motion estimation, all raw perfusion images are registered to the reference frame $\rho$ using 2D interpolation warping with the cumulative displacement computed from the stepwise estimates, which fully resolves the pixel-wise $x, y$ excursions from $\rho$ to a given frame $t$ :

$$
\left[\mathbf{F}_{\mathbf{t}}^{\mathbf{x}}, \mathbf{F}_{\mathbf{t}}^{\mathbf{y}}\right]= \begin{cases}\sum_{i=\rho}^{t-1}\left[\mathbf{f}_{\mathbf{i}}^{\mathbf{x}}, \mathbf{f}_{\mathbf{i}}^{\mathbf{y}}\right] & t>\rho \\ \sum_{i=t}^{\rho-1}\left[\mathbf{f}_{\mathbf{i}}^{\mathbf{x}}, \mathbf{f}_{\mathbf{i}}^{\mathbf{y}}\right] & t<\rho\end{cases}
$$

Despite the precautions outlined above to minimize flow field estimation errors, transient signal intensity changes from MR imaging related artifacts may induce false motion estimates and distort the signal intensity and appearance of the registered frames. These field distortions are generally characterized by large, focal variations in the intensity gradients of the $x$ and $y$ displacement components. The system handles these errors by automatically relaxing the LDOF parameters $\alpha$ and $\beta$ for the contentious pair of frames when anomalies are detected in the flow field. This correction stage eliminates possible field distortion artifacts, which can impact qualitative reading and quantitative analysis of the perfusion series.

\subsection{Registration to $P D$ images}

Once motion correction for the perfusion images is completed, they are further registered to the PD frames via a bridge image that encodes only the anatomical information of the perfusion series. This image is constructed with the minimum intensity projection of the motion corrected series as it is not influenced by ventricular contrast dynamics. Its photometric profile is first matched to the histogram of the mean PD image. The flow field mapping them together is then computed with the process outlined above using a high gradient consistency parameter $\gamma$. The resulting deformation estimation is finally applied to the motion corrected perfusion series to align it with the PD images.

\begin{tabular}{lllll}
\hline & $R^{2}$ & Mean & SD & Range \\
\hline \multirow{4}{*}{ AIF } & Raw & $0.987 \pm 0.009$ & $0.017 \pm 0.013$ & $0.107 \pm 0.088$ \\
& Moco & $0.993 \pm 0.005$ & $0.012 \pm 0.010$ & $0.083 \pm 0.074$ \\
& $p$ & $<0.001$ & $<0.05$ & $<0.01$ \\
\hline \multirow{4}{*}{ Myo } & Raw & $0.991 \pm 0.007$ & $0.012 \pm 0.009$ & $0.040 \pm 0.043$ \\
& Moco & $0.997 \pm 0.002$ & $0.003 \pm 0.004$ & $0.017 \pm 0.025$ \\
& $p$ & $<0.001$ & $<0.001$ & $<0.001$ \\
\hline \multirow{3}{*}{ PD } & Raw & $0.628 \pm 0.143$ & $0.192 \pm 0.081$ & $0.741 \pm 0.276$ \\
& Moco & $0.668 \pm 0.136$ & $0.169 \pm 0.081$ & $0.616 \pm 0.257$ \\
& $p$ & $<0.05$ & $<0.05$ & $<0.01$ \\
\hline
\end{tabular}

Table 1: AIF, myocardial (myo), and PD image correlation statistics. Values are mean $\pm \mathrm{sd}$.

\section{QUANTITATIVE EVALUATION}

Both rest and stress CMR perfusion studies of 47 patients, comprised of 282 myocardial and 94 AIF series, were included in this pilot study to evaluate motion correction performance of the proposed framework. The default LDOF parameters were set to: $\alpha=4.1, \beta=390$, and $\gamma=7.5$.

\subsection{Full-frame correlation}

As a measure of frame-to-frame appearance consistency, correlation coefficients $\left(R^{2}\right)$ were computed on sequential image pairs for both raw and moco series of the AIF and myocardium cohorts. The mean, standard deviation (SD), and absolute range of $R^{2}$ were obtained for each series. A non-parametric Mann-Whitney $U$ test revealed significant improvement of the moco over the raw series for each of these measures, as shown in Table 1. As a representative example, Figure 3 illustrates how the motion correction helps preserve appearance throughout an adenosine-induced stress series at all three slice locations. In the raw series, we note the periodic fluctuations in the correlations caused by myocardial displacement and the consequence of a respiration gasp at frame 35. Conversely, at all three slice locations, the moco series shows little frame-to-frame correlation variations and the respiration event is largely compensated.

\subsection{Perfusion series to $P D$ image registration}

The effectiveness of the perfusion series to PD image registration is demonstrated in Figure 2. As opposed to the raw image series, the non-linear deformation of the myocardium border is corrected as it is adequately aligned to the PD image throughout the full course of the dynamic contrast enhancement series. Notable motion correction is evident at late perfusion, where patient respiration is known to occur. Across the myocardial cohort, the moco series show significant alignment improvement to the PD images, as seen in Table 1, where the PD images were correlated with the first baseline perfusion frames.

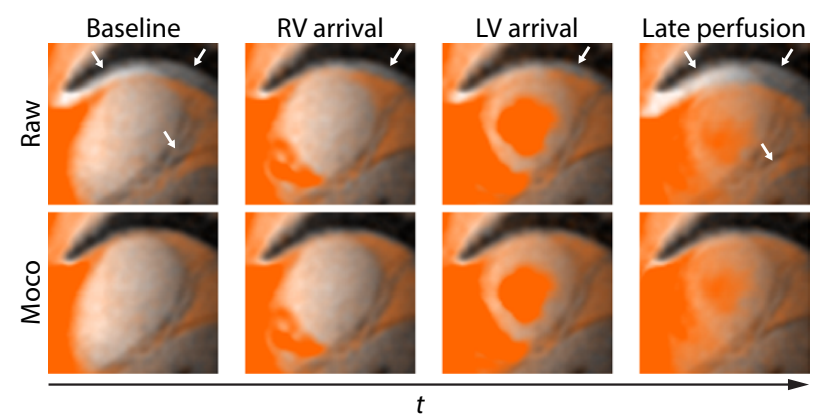

Fig. 2: Myocardial perfusion frames (orange) overlaid on a PD image (gray). Arrows indicate areas of large mis-alignment. Brightness and contrast adjusted to enhance structure visualization. 

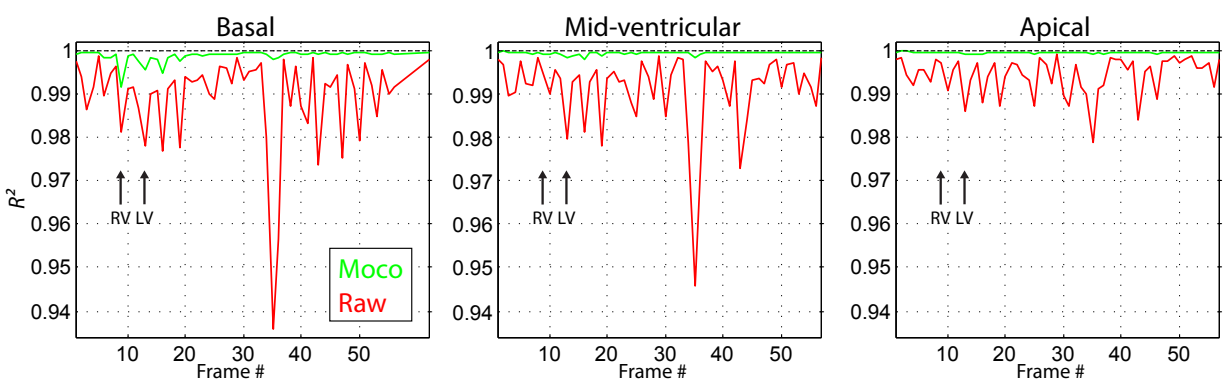

Fig. 3: Sequential frame correlation at different slice locations of a stress myocardial perfusion. Arrows indicate contrast arrival in the right and left ventricles.

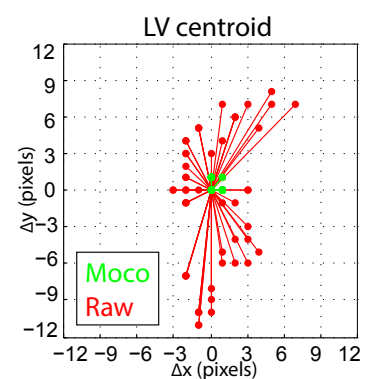

Fig. 4: Displacement map relative to the ref. frame.

\section{3. $L V$ centroid displacement}

Motion correction was evaluated locally in the heart region by computing the LV centroid displacement on the basal slices of each series. An ROI was manually positioned with a circular boundary around the LV at the peak enhancement frame and was then automatically propagated throughout the series with a correlation-based feature tracker. The ROI's centroid at each image was computed and used as an indication of the translational movement of the $\mathrm{LV}$ relative to the reference frame. This type of motion is largely corrected, as shown in Figure 4, where the moco series' residual movement is constrained to one pixel. The large vertical motion seen in the raw series is representative of diaphragmatic movement caused by respiration, which is also observed in Figure 2. Overall, the mean LV displacement is significantly reduced $(p<0.001)$ for the moco $(0.823 \pm 0.641$ pixels $)$ compared to the raw $(2.44 \pm 1.02$ pixels $)$.

\section{CONCLUSION}

The automatic nonrigid motion correction framework presented here was developed to handle multiple CMR image types which, coupled together, afford rigorous quantitative perfusion analysis. The use of LDOF motion estimation combined with post-hoc warping, error correction, and robust reference frame detection result in significant motion reduction across PD, AIF, and myocardial images. The multithreadable design reduces execution time and will scale favorably with newer processor architectures. The system has shown promising results and will be further tested under various clinical conditions as well as compared to other methods.

\section{REFERENCES}

[1] O.R. Coelho-Filho et al., "MR myocardial perfusion imaging," Radiology, vol. 266, no. 3, pp. 701-715, 2013.

[2] L.-Y. Hsu et al., "A quantitative pixel-wise measurement of myocardial blood flow by contrast-enhanced first-pass CMR perfusion imaging: microsphere validation in dogs and feasibility study in humans," JACC: Cardio. Imag., vol. 5, no. 2, pp. 154-166, 2012.
[3] V. Gupta et al., "Cardiac MR perfusion image processing techniques: A survey," Med. Imag. Ana., vol. 16, no. 4, pp. 767-785, 2012.

[4] N. Zarinabad et al., "Myocardial blood flow quantification from MRI-an image analysis perspective," Curr. Cardio. Imag. Rep., vol. 7, no. 1, pp. 1-9, 2014.

[5] G. Wollny et al., "Automatic motion compensation of free breathing acquired myocardial perfusion data by using independent component analysis," Med. Imag. Ana., vol. 16, no. 5, pp. 1015-1028, 2012.

[6] Li et al., "Pseudo ground truth based nonrigid registration of myocardial perfusion MRI," Med. Imag. Ana., vol. 15, no. 4, pp. 449-459, 2011.

[7] L. Tautz et al., "Phase-based non-rigid registration of myocardial perfusion MRI image sequences," in ISBI 2010. IEEE, 2010, pp. 516-519.

[8] J-P Thirion, "Image matching as a diffusion process: an analogy with maxwell's demons," Med. Imag. Ana., vol. 2, no. 3, pp. 243-260, 1998.

[9] H. Xue et al., "Unsupervised inline analysis of cardiac perfusion MRI," in MICCAI 2009., pp. 741-749. Springer, 2009.

[10] L.-Y. Hsu et al., "Surface coil intensity correction and non-linear intensity normalization improve pixelresolution parametric maps of myocardial MRI perfusion," in MICCAI 2003., pp. 975-976. Springer, 2003.

[11] P.D. Gatehouse et al., "Accurate assessment of the arterial input function during high-dose myocardial perfusion cardiovascular magnetic resonance," J. of Mag. Res. Imag., vol. 20, no. 1, pp. 39-45, 2004.

[12] T. Brox and J. Malik, "Large displacement optical flow: descriptor matching in variational motion estimation," PAMI, IEEE, vol. 33, no. 3, pp. 500-513, 2011.

[13] N. Dalal and B. Triggs, "Histograms of oriented gradients for human detection," in CVPR 2005. IEEE, 2005, vol. 1, pp. 886-893. 